\title{
Cinética de Autooxidación del Ascorbato por Iones Cúpricos
}

\author{
LUZMILA TRONCOSO, EMILIO GUIJA \\ Centro de Investigación de Bioquimica y Nurrición. Faculad de Medicina. \\ Universidad Nacional Mayor de San Maros.
}

\begin{abstract}
RISSUMEN
La autooxidación del ascorbato catalizada por Cua se realiza a travís de una réacción de primer orden, proceso que es dependiente de la concentración de $\mathrm{Cu}^{2+}$ y que oledecéa una cinćtica de saturación. Ėlo sugiere que en la secuencia catalítica sc formaría un complejo intermedio que limitaría la formación de los productos de la reacción, uno de los cuales es el radical libre hidroxilo. La tiourea inhibe notablemente la reacción de autooxidación, mientras que el glicerol no ejerce efecto alguno. Lil ascorbato y el Cu ${ }^{2+}$, utilizados en las concentraciones en que se produce la autouxidación, no escinden el pucute disulfuro del DTNB. I a histidina y el citrato inbiben tanto la reacción de autooxidación como la ruptura del enlace disulfuro del DTNB. Estos resultados sugieren cue la ruptura del pucnte disulfuro del DTNB se realizaría con intervención de los radicales libres hidroxilo, generados por el sistena ascorbato/Cu ${ }^{2+}, y^{\prime}$ paralelamente mediante un proceso de reducción o de hidrólisis.
\end{abstract}

Palahras claves: Ácido Ascórhico; Cobre; Radicales Lihres; Óxido-Reducción; Antioxidantes.

\section{KINITIC OF ASCORBATE-AUTOOXIDATION BY CUPRIC IONS SUMMARY}

A scorbate autooxidation catalized by $\mathrm{Cu}^{2+}$ occurs through a Cuta-concentration-dependent first order reaction, which fulfill saturation kinetic rules. It suggests that could be an intermedial conplex synthetized in the catalitic sequence, which probably limits the formation of reaction-products (v. g., free radical hydroxil, and oth(rs). Autonxidation reaction is strongly inhibitted by thiourea, while glicerol have vo effects on it. Disulfure-binding on DNTB was not broken by ascorbate and Cu ${ }^{2-2}$, used in the same concentrations which occurs in autooxidation process. Histidine and citrate inhibits both autooxidation reaction and DNOB-disulfure binding break. These results suggests that the DNTB-disulfure bindingbreak was uncdiated by Ascorbate/Cü2+ systeni-generated hydroxil free radicals, as well as through a reduction process or hydrolisis.

Key words: Ascorbic Acid; Copper; Free Radicals; Oxidation-Reduction; Antioxidants.

\section{INTRODUCCIÓN}

El ascorbalo es una vilamina que se encuentra ainpliamente distribuída en irutas y verduras (1.a), pero no

Correspondenria:

Dia. Lisnila Troncoso.

Centro de Imestigación de Bioquimica y Nutrición.

Fachultad de Medicina. U.N.M.S.M.

Apartado Poisal No 1546 Lima-Perú. es sintelizado por el hombre, por cuyo molivo es imprescindible que lo incluya en su dieta $\left(^{3}\right)$.

Existen diversos estudios que atribuyen al ascorbato un papel fundamental en las reacciones de hidroxilación, como un energico agence reductor biologico y con papeles bien definidos en la síntesis de carnitina y esteroides. la absorción de hierro no hem, la formación de colágeno, la función inmune y el melabolismo de nucleótidos cíclicos, aminoácidos, colesterol, glucosa yácido fólico $\left(^{4-7}\right)$. 
El ácido ascórbico en presencia de trazas de melales de transicion. como el $\mathrm{Cu}^{2+}$. genera radicales hidroxilo a través de un proceso de alutooxidatión ("s). La presencia de EDTA inhibe esta reaccion. debido a que forma un complejo con el $\left.\mathrm{Cu}^{=-}{ }^{8}\right)$. Cuando el ascorbato scencuentra en prescencia de una concentración micromolar de Cu**, incrementa considerablemente la oxidación de diversos compuestos biologicamente importantes. tales como las proténas ( $\left.{ }^{9-11}\right)$ : polisacáridos (i:) y ácidos nucleicos $(13.14)$.

La lipoperoxidación es estimulada cuando el ácido ascorbico se encuentra en presencia de sales de cobre o hierro, reacción que se produce con la lormación de especies reactivas como son los radicales libres hiaroxilo; pero en contraposición a ello, el ascorbato tienc la capacidad de conferir una cliciente protección contra la peroxidacion Iipídica (15.16) y en esc contexio pucde restaurar las propiedades antioxidantes de la vilamina E oxidada, lo que podría constiluirse en una de las runciones mas importantes de esta vilamina (1?). La formation de peróxida de hidrogeno que se observa en el ojo es muy probable que se cleve debido a la oxidación no enzimática del ascorbato $\left({ }^{13}\right)$.

El efecto que eventualmente puede ejercer el ascorbato es dependiente de su concentración. y en lal sentido puede comportarse como un eficiente antioxidante o en su defecto actuar como un prooxidante (10.20). Cuando las concentraciones de ascorbato se encuentran por cncima de $1 \mathrm{mmol}$ actúa como un prooxidance en presencia de melales de transicion, tales como $\mathrm{Fc}^{3+}$ y $\left.\mathrm{Cu}^{2-}(2) .22\right)$.

Esta vitamina tiene la propiedad de reducir los puenles disulfuro de compuestos de origen biologico. así como de aquellos obtenidos por síntesis. La reducción del ácido 5,5 ditiobis (2-nitrobem\%oico) (DTNB) por el sistema ascorbalo/C $u^{2+}$. se incrementa considerablemente en presencia de cistina (2-3), en cambio. la presenciá de superóxido dismulasia o catalasa ocasiona una disminución considerable de la rupura del puente disulfuro del DTNB $\left({ }^{8}\right)$. En el presente trabajo se describe el proceso cinćlico de allooxidación del ascorbato y la probabilidad de que la ruptura de puentes disulfuro se realice exclusivamente por acción de radicales libres.

\section{MATERIAI, Y MÉTODOS}

\section{Materiales}

En el manejo experimental se utilizaron ácido $5.5^{\circ}$ ditiobis (2-nitroben\%oico) (DTNB) e histidina (Sign)a
Chemical Company'). fostato monopolásico. glicerol, citrato. sulfato de cobre (E. Merck Darmstade), acido ascorbico y tiourca (Ricdel de Hacn). Todas las solucioncs se prepararon con agua bidestilada en vidrio.

\section{Métodos}

\section{Reacción de autooxidación}

Las soluciones de ascorbato y las de DTNB se prepararon inmediatamente previas a la ejecución de cadia experimento. La aulooxidación del ascorbalo se delermino midiendo la disminución de la absorbancia a 265 nm (:-4) utilizando para los cálculos un coeliciconte de extinción molar de $19.300 \mathrm{M}^{-1} \mathrm{~cm}^{-1}$, valor que obtuvimos experimental mente en mestro lahoratorio. El medio de reaceion contenia. en un volumen final de 3.0 miL: tampón lostato de potasio $50 \mathrm{mM}$. pH 8.0. ascorbalo $\left(3,3 \times 10^{-5} \mathrm{M}\right)$ y concentracioncs diversas de Cuñ $\mathrm{u}$ olras sustancias motivo de experincentación. La reación so realios a temperatura ambiente y se iniciómediante la adición del $\mathrm{Cu}^{z+}$. la modilicación de la absorbancia se midió en un gralicador acoplado a un espectrofotómeco Gillord modelo 240.

\section{Ruptura del enlace disulfuro}

El ácido 5.5' diuohis (2-nituohenzoico) es un compuesto que por escision de su puente disulfuro libera tionitroben\%oato que absorbe a $412 \mathrm{~nm}$ ("s). El medio de ensayo contenía en un volumen de $2.0 \mathrm{~mL}$. lampón lisslato de potasio $50 \mathrm{mM}$. pH 8.0, acido 5.5' ditiobis (2nitroben\%oico) 1 m $M$ y coneentraciones variables de ascorbato y Cü. La liberación del lionilrobenzoalo se midio a 412 nm en un espectrofinfometro Gilford modelo 240 acoplado a un graficador. y sc cuantifico utilizando un coeliciente de extincion molar de $136(x)\left(\mathrm{M}^{-1} \mathrm{~cm}^{-1}\right.$

\section{RISLLITAIOS}

El ascorbalo es una vilamina que en medio acuoso y en presencia de oxigeno sufre un proceso de atlooxidacion que es acelerado a $p \mathrm{H}$ alcalino por melales de ransicion. La allternidación del ascorbato se siguio midicndo la disminución (de la absorbancia a 265 nm en em medio de reación ajuslado a pH 8.() al que se le adicionó concentraciones dilerentes de $\mathrm{Fe}^{5-}$. Co $\mathrm{Co}^{2+} \mathrm{o}$ Cun La autooxidación del ascorbato en ausencia de los metales de transición antes mencionados. fuc muy baja.

En la Tabla $\mathrm{N}^{0}$ I se obscrva que el ascorbalo se aulooxida en presencia de C.1 ${ }^{2+}$ en $13 n$ grado notablemente mayor que cuando se ulilisaron los metales $\mathrm{Co}^{2+}$ 
o Fe $\mathrm{F}^{3+}$. los que, en concentraciones dos veces mayores que el $\mathrm{Cu}^{2+}$ : produjeron una muy discreta autooxidación del ascorbato.

Tabla $N^{0}$ 1.- Autooxidación del ascorbato en presencia de melales de transición. *

\begin{tabular}{ccc}
\hline Metal & Concentración & $(\mathbf{n m} / \mathrm{min})$ \\
$\mathrm{Cu}^{2+}$ & $33.33 \mu \mathrm{mol}$ & 35.17 \\
$\mathrm{Co}^{2+}$ & $66,30 \mu \mathrm{mol}$ & 0,83 \\
$\mathrm{Fe}^{3+}$ & $66.30 \mu \mathrm{mol}$ & 0,42
\end{tabular}

* El medio de ensavo contenia: rampón jossaro de poiasio 50 mM pH is, O, axcorbaro $33 \mu \mathrm{M}$ y los merales en las concentra. ciones indicadas.

El efecto ocasionado por el $\mathrm{Cu}^{2+}$ se pudo observar al utilizar concentraciones de este metal comprendidas entre 3,33 y $13,4 \mu \mathrm{M}$, este proceso se realiza a través de una reaccion de primer orden. Cuando se grafican las constantes de velocidad de primer orden en función de las concentraciones de $\mathrm{Cu}^{2+}$, se ohserva que esta dependencia no es lincal, sino que corresponde a una curva de naturaleza hiperbólica, resultado que sugiere una cinética de saluración, lal como se muestra en la Fig. $N^{\circ} 1$, donde es posible apreciar que el valor máximo de la concentracion de $\mathrm{Cu}^{2-}$ que hemos utilizado para calcular la constante de velocidad corresponde a 0,0134 mM. La regraficación de los resultados anteriormente oblenidos en doble recíproca permite obtener una recta, lo que indicaría que la generacion de radicales libres es salurable con respecto a la concentración de $\mathrm{Cu}^{2+}$. de lo que también se desprende el hecho que durante el proceso de autooxidación se forme un complejo intermedio.

Estos resultados se ajustarían a la siguiente ecuación:

\section{donde:}

$$
\frac{1}{k^{\prime}}=\frac{K d}{k_{1}[X]}+\frac{1}{k_{3}}
$$

$\mathrm{k}^{\prime}$ = constante de velocidad observada.

$\mathrm{Kd}=$ constante aparente de disociación del complẹjo intermedio.

$\mathrm{X}=$ complejo intermedio.

$k_{2}=$ comslante de velocidad de primer orden en la saturación.

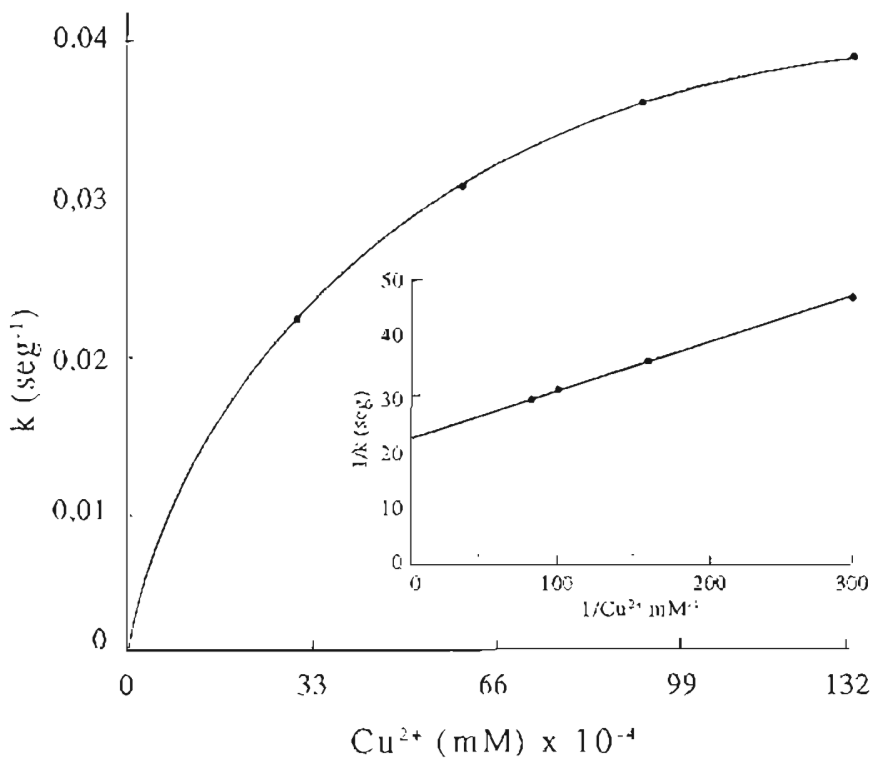

Fig. $\mathbf{N}^{\circ}$ 1.- Electo de la concentración de $\mathrm{Cu}^{2+}$ sobre la autooxidación del ascorbato. Medio de reacción: Tampón los a ato de potasio 50 m M pH 8:0, ascorbato 0,033 $\mathrm{mm}$ y $\mathrm{Cu}^{2+}$ en concentraciones variables.

El valor de la constante de velocidad de primer orden en la saturación es igual a $0,045 \mathrm{seg}^{-1}$ y fue calculada a partir del valor en el intercepto de la regralicacion en doble recíproca, mientras que la constante aparente de disociacion corresponde a $4,07 \times 10^{-3} \mathrm{M}$.

En el gráfico competitivo que se muestra en la Fig. $N^{0} 2$, se observa que la tiourca. compuesto que tiene la propiedad de captar radicales libres; inhibe Ia autooxidación del ascorbato, proceso que es lineal en las condiciones experimentales descritas, habiéndose calculado a partir del mencionado gráfico una constante de protección de $1.75 \times 10^{-4} \mathrm{M}$.

La uilización del glicerol con el mismo propósjto. permitió observar que la autooxidación del ascorbato en presencia de $\mathrm{Cu}^{2+}$ no sufre alteración alguna, tal como se muestra en la Figura $\mathrm{N}^{0} 3$. 


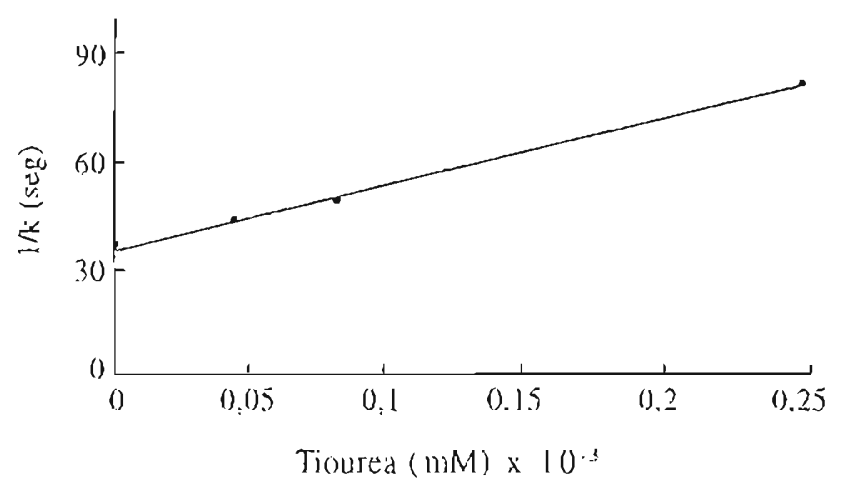

Fig. N"2.- Electo de la tiourea sobre la autooxidación del ascorbato calalizada por el $\mathrm{Cu}^{2+}$ Medio de reacción: Tampón fosfalo de potasio $50 \mathrm{mM} \mathrm{pH} \mathrm{8,0,} \mathrm{ascorbalo}$ $0,033 \mathrm{mM}$ y $\mathrm{Cu}^{2+} 0,0066 \mathrm{mM}$ y tiourea en diferentes concentraciones

Cuando se utilizaron concentraciones de $\mathrm{Cu}^{2+} 1,34 \mathrm{x}$ $10^{-5} \mathrm{M}$ y ascorbato $3.3 \times 10^{-5} \mathrm{M}$. que son las que se usaron para al proceso de athooxidación. no tue posible escindir el puente disulfuro del ácido $5,5^{\circ}$ diciobis (2nirrobenzoico) (DTNB): en cambio. si se incrementa la concencración de ascorbalo a 2,0 $\mathrm{mm}$ mantenjéndose la de $\mathrm{Cu}^{2+}$ a una concentración $0.01 \mathrm{mM}$ : la liberación de tionitrobenzodto: que es el compuesto que se produce conio consecuencia de la ruptura del puente disulfuro del DTNB. ocurre a una velocidad de $1,5 \mu \mathrm{M} / \mathrm{n}$ in.

Tabla N'2.- Efecto de inhibidores sobre los procesos de autooxidación y; ruptura de puentes disulfuro del

$$
\text { DTNB.* }
$$

\begin{tabular}{|c|c|c|}
\hline $\begin{array}{l}\text { Inhibidor } \\
(0,5 \mathrm{mmol})\end{array}$ & $\begin{array}{c}\text { Inhibición de la } \\
\text { autooxidación } \\
(\%)\end{array}$ & $\begin{array}{c}\text { Inhibición de la ruptura } \\
\text { de puentes disulfuro } \\
(\%)\end{array}$ \\
\hline Histidinat & 89.02 & 85.56 \\
\hline Citrate & $50 .(0)$ & 53.00 \\
\hline
\end{tabular}

* Las condicioner de ensayo se describen en Mélodos.
La presencia du histidina o citralo en el medio en que el ascorbato se autooxida por acción del $\mathrm{Cu}^{z-}$, ocasionó una notable inhibición, sicndo mayor el electo producido por la histidina. Un efecto similar se obtuvo cuando los compuestos anteriormente mencionados estuvicron presentes en una concontración (0.5 $\mathrm{mM}$ en el medio donde se produce la ruptura del puente disulfuro del DTNB por acción del sistema ascorbato/ $\mathrm{Cu}^{-}$.

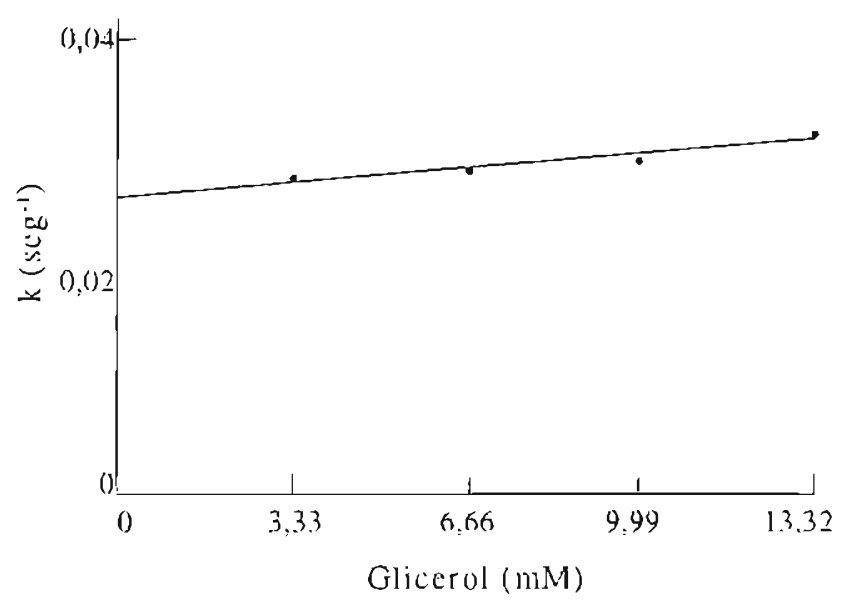

Fig. $N^{\circ}$ 3.- Efecto del glicerol sobre la autooxidación del ascorbato catalizada por $\mathrm{Cu}^{2+} \mathrm{Medio}$ de reaccion: Tampón fusfato de potasio $50 \mathrm{mM} \mathrm{pH} \mathrm{8,0,} \mathrm{ascorbato}$

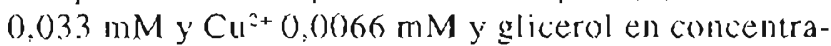
ciones variables.

\section{DISCUSIÓN}

Diversos estudios sobre la cinćtica de oxidación del ascorbato calalizada por cobre: permitieron formular inicialmente un mecanismo que incluye una interacción entre el ion ascorbato y el ion cúprico. hecho que lacilila la Iranslerencia interna de un electrón. El ion cuproso formado es oxidado por el oxígeno molecular. y la forma de seniquinona del ascorbato, por el oxígeno y los iones cúpricos (26). 
La alutooxidación del ascorbato por metales de transicion ha sido descrita por diversos autores ( $(7.2)$ ). este proceso se realizal conjuntamente con la reduccion del oxígeno molecular por un electrón y' la formación de radicales libres hidroxilo ( $\left.{ }^{s}\right)$. Los resultados mostrados en la Fig. $N^{\prime \prime} 1$ sugicren que en este proceso de aulooxidacion se formaria un complejo intermedio que limitaría dicha reacción, lo que está corroborado por la regraficación en deble recíproca.

Tambien se ha mostrado que la oxidación del ascorbato se estimula lig̣eramente en presencia de Fe"." reacción que muestra un marcado incremento cuando se adiciona EDTA. y es inhibida en lorna muy discrela por la superíxido dismutasa; estas observaciones permiten sugerir que el ascorhato es oxidado por el complejo EDTA/Fe't. lo qué estaría acorde con un incremento del potencial rédox del ion férrico cuando forma complejo con el EDTA (") milares a las observaciones descritas anteriormente: conlarmese aprecia en la Tabla $\mathrm{N}^{0}$ 1. la presencia de Fe $e^{3+} 0$ $\mathrm{Co}^{2+}$ ocasionó una discrela autooxidación del ascorbato. co comparación con aỵuella producida por el $\mathrm{Cu}^{2+}$.

Siendo el pKa del ascorbato 4.25, significa que a $\mathrm{pH}$ lisiológico la lorma predominante corresponde al ansón ascorbato, compuesto que oxidativamente formáácido dehidroascórbico, proceso que ocurriría mediante la oxidación reversible con la formación de un compuesto intermedio de radical ascorbilo ("ib). Las propiedades antioxidantes del ascorbato tienen relación con la haja reaclividad que tiene el radical semidehidroascorbato $\left.{ }^{31.3^{7}}\right)$.

El efecto ejercido por la tiourea en el sentido de inhibir la autooxidación del ascorbato por el $\mathrm{Cu}^{2+}$, probablemente haya sido ocasionado por la propicedad que tiene esce compucsto para ligarse a melales ("*), lambién existe la posibilidad de que la tiourea reacione con el peróxido de hidrógeno que se forma en la secuencia de reacciones que se produce durante la autooxidación del ascorbato, aunque no debe descartarse la alta afinidad que liene la liourea por el ion cuproso. cuya oxidacion permite la generación de radicales hidroxilo $\left({ }^{3 .}\right)$, resullado que es similar a la inhihición ejercida por estecompuesto sobrc la hidroxilatción del salicilato por acción del ascorbato, $C u^{i-} y^{\prime}$ peróxido de hidrógeno. El gráfico de competición lineal mostrado por la liourca, sugiere que este compuesto compite con el ascorbato en el pro- ceso antes descrito. en cambio el glicerol no ejerció efecto algumo, pero sí fue capay de inhibir considerablemente la ruplura del enlace disulluro del DTNB ("4).

La modificación selectiva de un residuo de histidina de la angiocensina 1, se produce en condiciones en las cuales el ascorhato reacciona con cantidades catalíticas de $\mathrm{Cu}^{2+}$. El daño oxidalivo que dicho sistema ocasionat al péptido se realiza específicanente en la posición C-2 del anillo imidazólico del residuo de histidina del péptido. Nuestros resultados tienen cierta correlación con los anteriormente descritos: yá que la presencia de histidina en el medio de reacción de autooxidación produce una nolable inhibicion del proceso. Tanto el ascorbato como el $\mathrm{Cu}^{2+} \mathrm{y}^{\prime}$ la histidina son compuestos cjue normalmente se encucntran on los alimemos y en las células del organismo $\left({ }^{i s}\right)$.

No ha sido posible encontrar evidencias de la formación del radical superóxido durante el proceso de autooxidación del ascorbato. la acción inhibitoria mostrada por la superoxido dismulasa era probablemente debida al efecto de la molécula proleica de la enzima y no a la remoción del radical superóxido $\left({ }^{36}\right)$.

Ciertos autores han indicado que la ruptura del puente

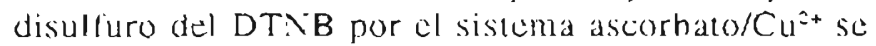
reatiza exclusivamente por acción de los radicales libres hidroxilo $\left({ }^{8}\right)$ : ya que en presencia de catalasa o manitol se produce una notable inhibición. El hecho que la ruptura del pucnte disulfuro del ácido 5,5' ditiobis (2-niarobenzoico) no ocurrden las mismas condiciones experimentales en que se aulooxida el ascorbato, permite sugerir que dicha escisión se produce no solamente por electo de los radiciales libres generados por el sistema ascorbato/ $\mathrm{Cu}^{2+}$. sino que debe operar otro mecanismo en forma paralela, tal como la reducción o la hidrólisis: lo que también estaría sustentado por el clecto inhibitorio que ejerce el glicerol sobre la ruptura del cnlace disulfurn del DTNB. asi como por el hecho de no alectar en forma alguna la autooxidación del ascorbato.

\section{AGRADIECINILNTO}

El presente trabajo lue linanciado por el FEDU (Fondo Esplecial de Desarrollo Universitario), Proyectos $\mathrm{N}^{\circ}$ $42400(05$ y 5240112 


\section{BIBLIOCRAFÍA}

1) Satuberlicla H. Connoimientos actusles sobre nutrición. 6" Fd. Wushington 1)C: OPS:ON:1S - ILSS: 1941. Publicación cicntifica $\$ 5.32: 152-162$.

2i Marks.J. The vitamins. The u role in Medical practice. I ancaster. Fingland: MTP Pressi ald Fillon Home; 1985. pp. 185-93.

3) Pauling L. A le-evaluation of vilamin C. En: Hanck A. Ril\%cl Cj. edilores: Re-evaluation of vitamin C. Nien. Suiza: Verlag Hans Huber Bern Stullgart: 1977.

4) Englard $S$ y coll. The biochemical functions of ascorhic acid Aisn Rev Nutr 1986: 6:36.5-406

5) Padh H. Vitamin C: Newer losightes into its bischemical functions. Nulr Rev 1991: 49(3): 65-70.

6) Jacob R. Assessment of huntan vilamin C status. J Nutr 1990: 120:1480-5.

7) Bates C. Function and melabolism of vitimin $C$ in man. En: "Vilamin (.". Lendrex: Applicd Science: 1981.

8) Inome H. Hirobe M. Disullide cluavige and insuline denaturalion hy active oxigen in the copper =1/Ascortic acid system. Chem Pharn Bull 1986: $34+3)$ : 1075.9.

9) Samuni A. Aromoviteh I y col. On the cyedexicity of vitamin C. and metisls ions. Fur I Birchem 198:3: 137:119-24.

10 shinar li, Navok T. Chevien M. The analogesus mechanismi of enzymalic inactivation induced hy ascoshate and superoxide in the presence of eopper. I Biol Chem 1983:258:14778-83.

11) Isbell H. Frush H. Oxidation of l. A worbic: preparation of L.Thronic ievid. Carh Res 1979: 72: 301-4.

12) Rofinson A. Reichheimer S. Instahility and lunctions: Ascorhic acid and glucaminyl and axparaginyl residucs. Am NY Acad Sci 1975: 258: 314-6

1.3) (hion S. DNA-and protein-scission activities of ascorbate. in lhe presence of copper jon or a copper-pepeide complex. J Biochem 1983: 94: 1259.67.

14) Aruona O, Halliwell li. Cajjesski ki, Dizdaroghu M. Copperiron dependent damage to llat hases in DNA in lle presence of hydrosen peroxide. Biochem I 1991: 273:2601-4.

15) Frei R. Ascorbic acid protects lipid in human plasma and LDL, against oxidative damage. Am J Clin Nutr 1991: 54(Suppl): $11135-11185$

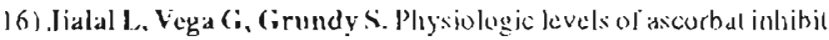
oxidative modilication ol low density lipoprolein. Alleresclerosis 1900: $82: 1 \times 5-91$

17) Witting I, Horwitt M. Filicul nl degree of latty acid unsalturation in locepherol deficiency-induces ereatininuria. I Nutr 1964: 82: 19.33.

18) Butetner G. Ascorbate aulexidation in the presence of iron and copper chelates. Free Rixd Rei Commun 1986: 1: 349-53.
19) Bendich A y col. Interaction of dietary Vitamin C and Vitamin $E$ on guinea pig immune responses to mirogens. I Nulr 1984: 114: $1.588-93$

20) Bendich $A$ et al. The antioxidant role ol vitamin C. Adv Free Rudical Biol Med 1986: 2: 419.44 .

23 just $S$, Morehouse $A$, Thomas $C$. Role of mals in exysen radical reactions. Free Radical Biol Med 1985: 1: 3-25.

22) (Girotti A. Mechanisms of lipid peroxidation. Free Radical Biol Med 1985: 1: 87-95.

23) Kloty I. Camplicl R. Copper-induced hydrolysis of the disullide bond. Arch Binchem Bioploys 1962: 96: 92-9.

24) Lewin $S$, Vitamin $C$ : Its molecular hiology and medical potenlial. Nueva York: Aciklemic Press: 1976. pp. 6-9.

25) lillman (i. Tissue sulphydryl groups. Arch Binchem Biophys: 1950): 82: 70.8 .

26) Weissberger $A$ y col. Oxidation processes. XVI. The autoxidation of ascorhic acid. J Am Chom Sac 194.3: 65: 19.34-9.

27) Khan M.1. Mariell A. Malal ion and melal chelate calalyou oxidation of ascorbic acid by molecular oxygen. I. Cupnic and furic ion calalyzed oxidation. J An Chem Soc 1967: 89(16): $4176-85$

28) Khan M. Martell A. Kinclics of metal ion and melal chelitte caldy\%el oxidation ol axcorbic acid. חI. Vanalyt ion caltaly\%ed oxidation. I Ain (hem Soc 1968:90(22): 6011-7.

29) Klein S. Cohen G. Cederhaum A. Production of formaldelyde during metaholism of dimethyl sulfoxide hy hydenxyl radical generating systems. Binclsem 1981: 20: 6006-12.

30) Laroff ( $i$, k reesenden $\mathrm{K}$, Schuler $\mathrm{K}$. The electron spin sesonance spectra of radical infermediates in the oxidation of ascorbic acid ald related substances. J Am Chem Soe 1972; 94(26): 9(062-73.

31) Cábelli D. Biclski B. Kinelics and mechanlism for de nxidution of

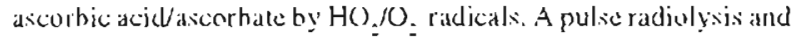
stopped llow photolysis study. J Phys Chem 1983:87: 1809-12.

32) Cederbaum $\mathrm{A}$ et al. Eifect of thiourea on microsomal oxidation

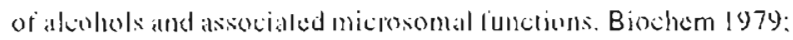
18: 1187-91.

33) Butt V. Hallaway M. The catily'sis of axcorbate oxidation by ion cupper and its complexes, Arcls Biechem Biophys 1961: 92: 24-32.

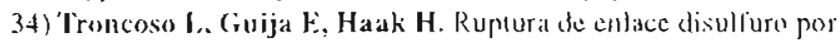
el sistema Axcolbato-EIJTA. Bol Soc Quim Perú 1994: 60)(1): $78-85$.

35) Uchida K. Kawatishi S. Sik-npecitucaxidation of angiolensin l hy copper" and l.-ascorbate: conversion ol histikline residues to 2-imidizolnnes. Arch Bieclem Biuphyss 1990: 283: 20-6.

36) Haliwell b. Foyer C. Ascurtic acid. melal inns and the superoxicle radical. Binchem I 1976: 155:697.7(30)

37) Gujja liy Troncoso L. Vilamina C': algunos roles insporlantes. Ker Acad l'cr Farm 1995: 10 (1): 52-6. 